\title{
Frequency-domain mesh-free finite-difference operator for visco-acoustic wave equation
}

\author{
Junichi TAKEKAWA $^{1}$ and Hitoshi MIKADA ${ }^{1}$ \\ ${ }^{1}$ Dept. of Civil and Earth Res. Eng., Kyoto University
}

\begin{abstract}
In the present study, we apply a mesh-free finite difference method (MF-FDM) to frequency-domain visco-acoustic wave propagation. Although the full-waveform inversion (FWI) is a powerful tool to investigate the subsurface quantitatively, FWI requires a huge amount of computer resources. This poses an obstacle to apply FWI to practical studies especially in three-dimensional cases. MF-FDM has a potential to decrease the computational costs for making synthetic data sets in a simple manner. We demonstrate the effectiveness of MF-FDM using numerical experiments. Our results show that the proposed method can improve the numerical efficiency without sacrificing the numerical accuracy.
\end{abstract}

\section{INTRODUCTION}

Recently, the full-waveform inversion (FWI) has drawn attention in scientific and engineering fields because of its strong possibility to extract quantitative information of the subsurface ${ }^{1)}$. One of the main problems of FWI is high computational costs. Especially in the frequency-domain FWI, we have to solve linear equations to make synthetic data sets. This poses an obstacle to apply FWI to real practices in three-dimension. To develop a novel method for reducing the CPU time and required computational memory is a solid solution to this problem.

A number of numerical methods to solve the wave propagation have been developed ${ }^{2,3}$. Almost all of techniques are base on the finite-difference method (FDM). On the other hand, mesh-free methods attract attention as alternatives to the conventional finite-differences because of its high flexibility of discretization. Recently a mesh-free finite-difference method (MF-FDM) has been proposed by Takekawa et al. $(2015)^{4)}$. The spatial resolution is locally refined to reduce the number of calculation points without sacrificing the numerical accuracy in a simple manner. This method works well to reduce the computational costs especially in models with high velocity contrasts ${ }^{5}$. Although the effectiveness of the method has been demonstrated in the time-domain modeling, its ability in the frequency-domain modeling has not been revealed yet.

In the present study, we apply the mesh-free method $^{4)}$ as a simulator in the frequency-domain modeling. To evaluate the effectiveness of the
MF-FDM, we conduct a numerical experiment using a low velocity anomaly model.

\section{METHOD}

We briefly explain the visco-acoustic wave equation and the MF-FDM.

\section{(1) Visco-acoustic wave equation}

We solve the Helmholtz equation with the attenuation term as follows.

$$
\left(v^{2}+i \omega \eta\right)\left(\frac{\partial^{2} p}{\partial x^{2}}+\frac{\partial^{2} p}{\partial z^{2}}\right)+\omega^{w} p=0
$$

where $\mathrm{i}$ is the imaginary unit, $\omega$ is the angular frequency, $\mathrm{p}$ is the pressure, $\mathrm{v}$ is the velocity, $\eta=v^{2} /(Q \omega), Q$ is the quality factor. We discretize the equation using an operator of the MD-FDM as shown below.

\section{(2) Mesh-free FDM}

The mesh-free concept is based on the multi-variable Taylor expansion. The multi-variable Taylor expansion of a scalar function $f(r)$ to M-th order at position $\mathbf{r}_{\mathbf{i}}$ is expressed as follows:

$$
\begin{aligned}
& \mathrm{f}\left(\mathbf{r}_{\mathrm{i}}+\Delta \mathbf{r}\right)=\mathrm{f}\left(\mathbf{r}_{\mathrm{i}}\right)+\sum_{\mathrm{m}=1}^{\mathrm{M}}\left\{\frac{1}{\mathrm{~m} !}(\Delta \mathbf{r} \cdot \nabla)^{\mathrm{m}} \mathrm{f}\left(\mathbf{r}_{\mathrm{i}}\right)\right\}+ \\
& \mathrm{O}\left(\|\Delta \mathbf{r}\|^{\mathrm{M}+1}\right)
\end{aligned}
$$

where $\mathbf{r}_{\mathrm{i}}$ and $\mathbf{r}_{\mathrm{i}}+\Delta \mathbf{r}$ are the position vectors of grid $\mathrm{i}$ and its neighboring grid $\mathrm{j}$, respectively. In two-dimensional case, differential operators are calculated as follows: 
$(\boldsymbol{\delta} \mathbf{f})_{\mathbf{r}=\mathbf{r}_{\mathrm{i}}} \approx\left\{\sum_{\mathrm{j}=1}^{\mathrm{n}}(\mathbf{P} \otimes \mathbf{P})\right\}^{-1} \cdot\left\{\sum_{\mathrm{j}=1}^{\mathrm{n}}\left(\mathbf{P} \Delta \mathrm{f}_{\mathrm{ij}}\right)\right\}$

$\mathbf{P}=\left(\Delta \mathrm{r}_{1}, \Delta \mathrm{r}_{2}, \frac{1}{2 !} \Delta \mathrm{r}_{1}^{2}, \Delta \mathrm{r}_{1} \Delta \mathrm{r}_{2}, \frac{1}{2 !} \Delta \mathrm{r}_{2}^{2}, \cdots, \frac{1}{\mathrm{M} !} \Delta \mathrm{r}_{2}^{\mathrm{M}}\right)^{\mathrm{T}}$

$\boldsymbol{\delta}=\left(\frac{\partial}{\partial \mathrm{r}_{1}}, \frac{\partial}{\partial \mathrm{r}_{2}}, \frac{\partial^{2}}{\partial \mathrm{r}_{1}^{2}}, \frac{\partial^{2}}{\partial \mathrm{r}_{1} \partial \mathrm{r}_{2}}, \frac{\partial^{2}}{\partial \mathrm{r}_{2}^{2}}, \cdots, \frac{\partial^{\mathrm{M}}}{\partial \mathrm{r}_{2}^{\mathrm{M}}}\right)^{\mathrm{T}}$

where $f_{i j}=f_{j}-f_{i}$ is the difference between scalar values at positions $\mathbf{r}_{\mathbf{i}}$ and $\mathbf{r}_{\mathbf{j}}$. The details of the derivation of the above equations can be found in a reference ${ }^{4)}$.

As an absorbing boundary condition (ABC), we apply a hybrid technique for the frequency-domain modeling ${ }^{6,7)}$. For the hybrid ABC, we apply a buffer zone in the vicinity of model edges. In the buffer zone, coefficients of a linear system are determined by a weighted average of the one-way and two-way wave equations. Details can be found in a reference ${ }^{6}$.

\section{NUMERICAL RESULTS}

In this chapter, we quantitatively evaluate the computational costs of the method with and without the local refinement strategy.

\section{(1) Numerical model}

Figure 1 shows a numerical model of the present study. The model includes a low velocity anomaly in the center of the model. The anomaly has a P-wave velocity of $1500 \mathrm{~m} / \mathrm{s}$, whereas the back ground velocity is $3000 \mathrm{~m} / \mathrm{s}$. Q-vaule is set to 10 . The source and receiver array are located at the left

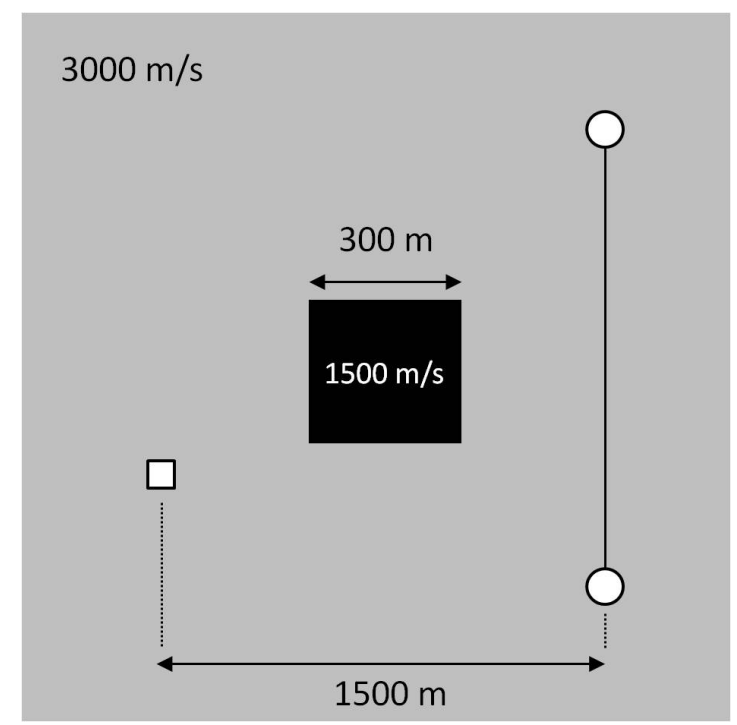

Figure 1: Numerical model with a low velocity anomaly. Open square and circle represent the source and the receiver locations, respectively.

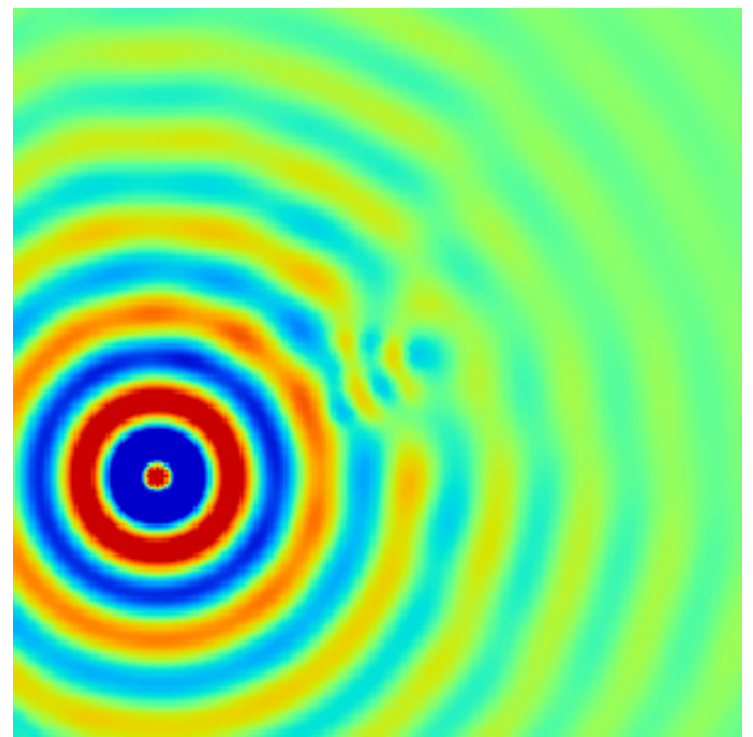

Figure 2: Monochromatic wavefield with $10.3 \mathrm{~Hz}$. 


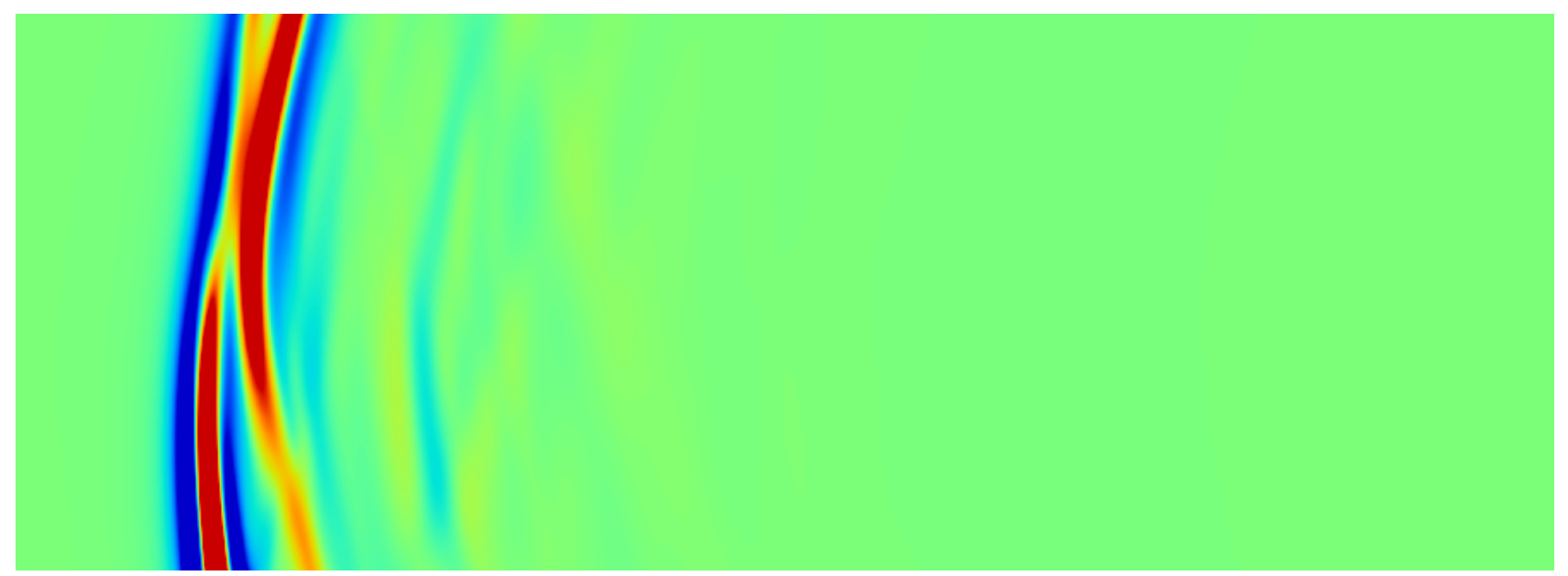

Figure 3: Shot gather obtained by the receiver array.

and right side of the anomaly, respectively. The source function is the Ricker wavelet with a central frequency of $10 \mathrm{~Hz}$. We use $10 \mathrm{~m}$ lattice structure for background, whereas $5 \mathrm{~m}$ interval is applied around the low velocity anomaly.

\section{(2) Wavefield and waveforms}

Figure 2 shows a monochromatic wavefield with the harmonic frequency of $10.3 \mathrm{~Hz}$. It can be seen that the wavefield is disturbed by the low-velocity anomaly.

Figure 3 shows a shot gather obtained by the receiver array. We can observe that the waveform can be calculated accurately without numerical dispersion especially behind the low velocity anomaly.

\section{CONCLUSIONS}

We apply the MF-FDM to the visco-acoustic wave propagation in the frequency-domain. We confirm the effectiveness of the method using an inhomogeneous medium with a low velocity anomaly. Our results show that the proposed method has a potential to be an alternative to the conventional FDM due to its computational efficiency.

ACKNOWLEDGMENT: This work was partly supported by MEXT/JSPS KAKENHI Grant Number 15K18301.

\section{REFERENCES}

1) Virieux, J. and S. Operto, 2009, An overview of full-waveform inversion in exploration geophysics, Geophysics, 74, 6, WCC127-WCC152.

2) Virieux, J., 1986, P-SV wave propagation in heterogeneous media: Velocity-stress finite-difference method, Geophysics, 51, 889-901.

3) Levander, A. R., 1988, Fourth-order finite-difference
P-SV seismograms, Geophysics, 53, 1425-1436.

4) Takekawa, J., H. Mikada, and N. Imamura, 2015, A mesh-free method with arbitrary-order accuracy for acoustic wave propagation, Computers \& Geosciences, 78, 15-25.

5) Takekawa, J. and H. Mikada, 2015, An adaptive resolution full-waveform inversion using a mesh-free finite-difference method, Proceedings of The 19th International Symposium on Recent Advances in Exploration Geophysics (RAEG 2015). doi: 10.3997/2352-8265.20140183

6) Ren, Z. and Liu, Y., 2013, A hybrid absorbing boundary condition for frequency-domain finite-difference modeling, Journal of Geophysics and Engineering, 10, 054003.

7) Takekawa, J. and H. Mikada, 2016, An absorbing boundary condition for acoustic wave propagation using a mesh-free method, Geophysics, in press. 